University of Wollongong

Research Online

Faculty of Informatics - Papers (Archive)

Faculty of Engineering and Information

Sciences

17-10-2007

\title{
Viability of Concurrent Transmission and Reception for UWB Radios over Multipath Channels
}

Ricardo Gandia Sanchez

University of Wollongong, rgs653@uow.edu.au

Xiaojing Huang

University of Wollongong, huang@uow.edu.au

K. Chin

University of Wollongong, kwanwu@uow.edu.au

Follow this and additional works at: https://ro.uow.edu.au/infopapers

Part of the Physical Sciences and Mathematics Commons

\section{Recommended Citation}

Gandia Sanchez, Ricardo; Huang, Xiaojing; and Chin, K.: Viability of Concurrent Transmission and Reception for UWB Radios over Multipath Channels 2007.

https://ro.uow.edu.au/infopapers/657

Research Online is the open access institutional repository for the University of Wollongong. For further information contact the UOW Library: research-pubs@uow.edu.au 


\title{
Viability of Concurrent Transmission and Reception for UWB Radios over Multipath Channels
}

\author{
Abstract \\ Multihop transmissions over wireless mesh networks present limited end-to-end (E2E) data rates, as \\ every hop waits for an entire packet to arrive before starting retransmission. That is, the E2E data rate \\ drops when every hop stores and then forwards packets, thus making such transmissions unsuitable for \\ multimedia applications. In this work, in order to increase data rates, we present an ultra-wideband (UWB) \\ radio transceiver capable of concurrently transmit and receive (cTxRx) packets. However, concurrently \\ receiving and transmitting comes at the cost of a channel model with increased interference level. Herein, \\ we explain a new interference model and propose a transceiver that compensates for it. We describe the \\ transceiver mathematically and analyze its performance via simulations. Also, we demonstrate that the \\ E2E data rate exceeds that of current multihop transmissions, thus allowing multimedia traffic to be \\ transmitted over a multi-hop wireless mesh network.

\section{Disciplines} \\ Physical Sciences and Mathematics

\section{Publication Details} \\ This conference paper was originally published as Sanchez, RG, Huang, X, Chin, K, Viability of Concurrent \\ Transmission and Reception for UWB Radios over Multipath Channels, International Symposium on \\ Communications and Information Technologies ISCIT 2007, 17-19 Oct, 1241-1246.
}




\title{
Viability of Concurrent Transmission and Reception for UWB Radios over Multipath Channels
}

\author{
Ricardo Gandía Sánchez, Xiaojing Huang and Kwan-Wu Chin \\ School of Electrical, Computer and Telecommunications Engineering \\ University of Wollongong \\ Wollongong, NSW 2522, Australia \\ Email: ricardo@titr.uow.edu.au, \{huang, kwanwu\}@uow.edu.au
}

\begin{abstract}
Multihop transmissions over wireless mesh networks present limited end-to-end (E2E) data rates, as every hop waits for an entire packet to arrive before starting retransmission. That is, the E2E data rate drops when every hop stores and then forwards packets, thus making such transmissions unsuitable for multimedia applications. In this work, in order to increase data rates, we present an ultra-wideband (UWB) radio transceiver capable of concurrently transmit and receive (cTxRx) packets. However, concurrently receiving and transmitting comes at the cost of a channel model with increased interference level. Herein, we explain a new interference model and propose a transceiver that compensates for it. We describe the transceiver mathematically and analyze its performance via simulations. Also, we demonstrate that the E2E data rate exceeds that of current multihop transmissions, thus allowing multimedia traffic to be transmitted over a multi-hop wireless mesh network.
\end{abstract}

\section{INTRODUCTION}

In a typical wireless mesh network, the store-and-forward $(\mathrm{S} \& \mathrm{~F})$ paradigm is used by each node to relay packets to their destination. This means each node needs to receive an entire packet correctly before transmitting it to the next node.

The main drawback of the store-and-forward paradigm is that the end-to-end (E2E) data rate decreases linearly with increasing hops [1] [2]. To overcome this drawback, the authors of [3] and [4] have proposed to increase the single-hop data rate. However, their solutions do not solve the drawback since the E2E data rate continues to reduce linearly with hops. Ramanathan [2] proposed an alternative paradigm whereby each relay node uses a distinct frequency to receive and transmit, thus enabling concurrent transmission and reception. Unfortunately, his approach increases radio complexity and cost.

In light of the aforementioned deficiencies, we propose an alternative model that allows nodes to concurrently transmit and receive (cTxRx) packets. The model is inspired by Huang [1] [5] where the single-hop data rate is maintained throughout the E2E transmission. In particular, we enable the radio at each node to concurrently transmit and receive using novel baseband algorithms that have no significant impact on the transceiver's complexity and cost. Adding to the proposed model, this paper contributes with a new channel model that characterizes local and remote interferences resulting from cTxRx. Our results confirm the viability of the cTxRx mode in multipath channels and we find that the E2E data rates obtained outperformed transceivers working in S\&F mode.
The paper is structured as follows. First, we outline the challenges to achieving cTxRx followed by a description of the channel model. Then in Section III, we describe the UWB transceiver mathematically and highlight cancellation techniques that can be used to overcome interferences. The implementation details of the transmitter, receiver and PHY layer packet structure are explained in Section IV, with Section $\mathrm{V}$ evaluating the transceiver's performance over multipath channels. The expresions for the E2E data rate under cTxRx and S\&F relaying scenarios are derived in Section VI. Finally, Section VII summarises our findings.

\section{Channel Overview}

Figure 1 illustrates a typical transmission pipeline over a linear topology with two relay nodes. Notice that all nodes are transmitting the same packet simultaneously, where for example node-A could be transmitting the end of the packet while node- $\mathrm{C}$ is starting to transmit its beginning.

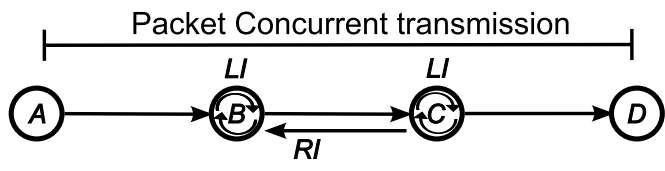

Fig. 1. Wireless mesh network operating in cTxRx mode with Local Interference (LI) and Remote Interference (RI) jamming node-B.

The above transmission pipeline faces a number of challenges with regards to packet reception. In particular, there are two interfering signals. The first one is a consequence of the power leakage that occurs due to the lack of isolation in radio hybrids. We deem this effect Local Interference (LI), as it is generated within the transceiver. In Fig. 1, the relay nodes, B and $\mathrm{C}$, have to compensate for LI as they transmit and receive the packet. The second one arises from the transmission of the next-hop node. That is, assuming omni-directional antennas, node-C's transmission reaches node-B with the same power level as node-A's transmission. Hence, both signals interfere at node-B. We deem this effect Remote Interference (RI), as it is generated by a remote node's transmission. In Fig. 1, the worst case scenario is represented by node-B, where both LI and RI are present.

Another challenge is the effect of interference over the Automatic Gain Control (AGC) circuitry. The AGC regulates the 
gain of the Low Noise Amplifier (LNA) in order to maximize the dynamic range of the Analog to Digital Converter (ADC). When higher power levels arrive due to interference, the AGC reduces the gain of the LNA, which in turn, decreases the resolution of the ADC. With decreased resolution, the received signal is sampled into a narrow range of values, thus losing vital information.

On top of interference, we need a channel model that integrates multipath and propagation components. In this respect, the multipath is implemented using the modified version of the Saleh-Valenzuela model proposed by the IEEE802.15.3 working group [6]. Also, in this channel model, both the transmitted signal and RI traverse different multipath channels, which complicates RI cancellation. The attenuation due to propagation is a calculated using the Friis formula,

$$
P L(d B)=20 \cdot\left(\log _{10}(d)+\log _{10}(f)\right)-K
$$

where $d$ is the distance between nodes, $f$ the central frequency of the transmission, and $K$ a constant dependent on the antenna design. Assuming that all antennas have a gain equal to 1 , then $K=-147.56 d B$. Given that we assume that all transmitting nodes are equidistant, both signal and RI present the same attenuation. On the other hand, at best, the LI will be attenuated by $20 \mathrm{~dB}$ traversing the radio hybrid.

\section{Transceiver Mathematical Description}

The transceiver is built around the MultiCode Interleave Direct Sequence (MCIDS) technology [7], which uses a set of $N$ sequences of length $P$. The data symbols are grouped in blocks of $N$ symbols, $a_{i}$, with $i=0,1, \ldots, N-1$. Each symbol is spread by the corresponding $i_{t h}$ sequence in the set. The processing gain is $P+P_{0}$, which includes a cyclic prefix (CP) of length $P_{0}$. We denote the code sequences as $c_{i}[j]_{P}$, with $j=-P_{0}, \ldots, 0,1, \ldots, P-1$.

Eq. (2) describes the transmitted baseband signal, where $T_{c}$ is the chip time and $p(t)$ is the impulse response of the pulse shaping filter stage prior to transmission.

$$
s(t)=\sum_{i=0}^{N-1} a_{i} \sum_{j=-P_{0}}^{N-1} c_{i}[j]_{P} p\left(t-i T_{c}-j N T_{c}\right)
$$

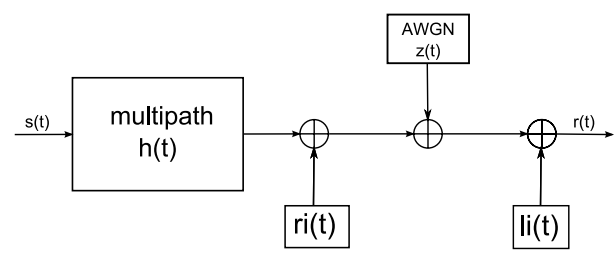

Fig. 2. Block model for the cTxRx Channel. $r i(t)$ and $l i(t)$ represent RI and LI resp.

Figure 2 describes a transmission signal, $s(t)$, over a cTxRx channel. The expresion for the received signal, $r(t)$, is shown in (3), where $z(t)$ is additive white Gaussian noise (AWGN) with spectral density $N_{0}$.

$$
\begin{aligned}
& r(t)=\rho s(t) \otimes h(t)+\rho_{r i} r i(t)+\rho_{l i} l i(t)+z(t) \\
& h(t)=\sum_{l=0}^{L-1} \alpha_{l} \cdot \delta\left(t-l T_{c}\right) \\
& r i(t)=s\left(t-T_{r i}\right) \otimes h_{r i}(t) \\
& l i(t)=s\left(t-T_{l i}\right)
\end{aligned}
$$

The parameters $\rho, \rho_{r i}$ and $\rho_{l i}$ are attenuation factors. The first two represent the attenuation, due to the propagation over the channel, of the signal and RI. The last one is the LI attenuation through the hybrid. The multipath channel, $h(t)$, is described in (3a) as a tapped delay line with $L$ paths. The channel coefficients, $\alpha_{l}$, are complex-valued independent random Gaussian values with Rayleigh distributed magnitudes and uniformly distributed phases.

The expresion for $r i(t)$, Eq. (3b), is a delayed version of $s(t)$, which faces a different multipath channel. On the other hand, the expresion for $l i(t)$ is a delayed version of the transmitted signal shown by (3c). Its delay, $T_{l i}$, is designed to minimize interference, which we will explain further in Section IV-C.

The received signal, Eq. (3), goes through a matched filter with impulse response $p(-t)$. To simplify its derivation, we assume that the combination of AGC/LNA/ADC circuitry is ideal, i.e., infinite dynamic range. Hence, the resulting baseband signal from the matched filter is presented by (4).

$r[n]=\int_{-\infty}^{\infty} r\left(n T_{c}+t\right) p(t) d t=r_{o}[n]+r_{l i}[n]+r_{r i}[n]+Z_{n}$

To gather all energy due to multipath, the despreading is embodied as a RAKE structure with L fingers, followed by Maximum Ratio Combining (MRC). Due to the use of a cyclic prefix, the correlations are circular, meaning that they wrap around the MCIDS block. Hence, the use of the modulo operation over the length of the MCIDS block, $P N$, is used in (5).

$$
r_{i, l}=\sum_{n=0}^{P N-1} r\left[(n+l)_{P N}\right] d_{i}[n]
$$

The circular correlations despread the baseband signal using the $d_{i}$ code sequences described by (6). The despreading sequences account for the interleaving action by introducing zeros between their base elements, $c_{i}[j]$, which creates a sequence of the same size as an MCIDS block. In short, they are built to simultaneously despread and deinterleave during the correlation operation.

$$
\begin{aligned}
d_{i}[n]=\sum_{j=0}^{P-1} c_{i}[j] \delta(n-i-j N) ; \quad & i=0, \cdots, N-1 \\
n & =0, \cdots, P N-1
\end{aligned}
$$

The decision variable, $U_{i}$, is calculated by applying MRC to the RAKE output. As it is observed in (7), both $U_{i, r i}$ and 
$U_{i, l i}$ denote the interference to cancel. The parameters $b^{r i}$ and $b^{l i}$ are their respective data symbols. The function $q(0)$ is the ideal Nyquist pulse $q(\tau)=\int_{-\infty}^{\infty} p(t+\tau) p(t) d t . \phi_{h h^{\prime}}$ and $\phi_{c_{i} c_{k}}$ are respectively the channel and the code correlation functions for RI.

$$
\begin{gathered}
U_{i}=\operatorname{Re}\left[\sum_{l=0}^{L-1} \alpha_{l}^{*} r_{i, l}\right] \\
=\operatorname{Re}\left[U_{i, r o}+U_{i, r i}+U_{i, l i}+U_{i, Z_{n}}\right] \\
U_{i, r i}=q(0) \sum_{k=0}^{N-1} b_{k}^{r i} \sum_{n=-P_{0}}^{P_{0}} \phi_{h h^{\prime}}\left(i-k-n N-\frac{T_{r i}}{T_{c}}\right) \phi_{c_{i} c_{k}}(n) \\
U_{i, l i}=q(0) \sum_{l=0}^{L-1} \alpha_{l}^{*} \sum_{n=-P_{0}}^{P_{0}} b_{l+i-n N-\frac{T_{l i}}{T_{c}}}^{l i} \phi_{c_{i} c_{m}}(n)
\end{gathered}
$$

The term $U_{i, r i}$ is zero when $k \neq i$. In other words, assuming perfect orthogonal codes, RI is forced to interfere with a different sequence other than the one used to code it. This is achieved by manipulating the retransmission delay, $T_{r i}$. More details are given in Section IV-C.

Similarly, the term $U_{i, l i}$ is zero when $m \neq i$. A paralell strategy could be used to cancel LI. However, the difference in power levels makes code orthogonality very critical. As there is no perfect code, other approaches have to be considered. We describe them in Section IV-C.

\section{System Design}

\section{A. Transmitter}

MCIDS produces a baseband signal designed to provide sufficient processing gain to meet the required transmission range between hops. The spreading sequences used are derived from a Hadamard matrix of order 16. This produces a code set of 16 orthogonal sequences of length 16 . The Hadamard sequences have good cross-correlation properties to help combat ISI in an efficient manner. Also, a cyclic prefix of length 1 is added to further improve ISI resilience.

The first step performed by the transmitter is to modulate the incoming bits using BPSK, QPSK, 16QAM or 64QAM depending on the required transmission rate. Once the symbols are created, the second step is to feed them into the MCIDS stage for spreading. Although MCIDS can spread 16 symbols in a block, only 12 symbols are processed at a time. The other 4 symbols are used as pilots to estimate the channel if required. Here, despite the pilots being kept in the MCIDS blocks, we assume that the channel is known. Finally, interleaving allows transmissions over channel lengths of 16 MCIDS chips, or $100 \mathrm{~ns}$ with $T_{c}=6.25 \mathrm{~ns}$.

The transceiver works in either S\&F or cTxRx. For cTxRx, the next step is to duplicate the MCDIS blocks according to an Outer Code (OC), which enables the receiver to remove LI. The role of OCs is explained in more detail in Section IV-C. The OC step is skipped when the radio operates in S\&F mode, given that there is no LI to cancel.
After the OC step, the baseband signal is fed at a rate of 16 samples per chip into a pulse-shaping second order Butterworth filter with a $-3 \mathrm{~dB}$ cutoff frequency of $0.64 \mathrm{GHz}$. The filter produces a pulse width in the range of 1 to $2 \mathrm{~ns}$, thereby generating a $1 \mathrm{GHz}$ UWB signal that is compliant with the FCC spectral mask. Finally, the signal is transmitted using an ideal broad antenna.

\section{B. Receiver}

At the receiver, the signal is fed into a matched filter to improve its SNR. Then, it is amplified by the AGC/LNA circuitry and is sampled at the rate of $\frac{4}{T_{c}}$. During cTxRx, the AGC is only enabled during the packet preamble; it is switched off at other times. This avoids the AGC reajusting to LI power levels, which prevents the radio from receiving the signal completely.

The baseband signal is then despread using a 32 finger RAKE architecture of circular correlators following MRC to yield the decision variables. If the transceiver operates in cTxRx, the despreading of the OC is performed before the RAKE.

\section{Interference Cancellation Algorithms}

In order to cancel RI and LI, we propose two cancellation algorithms that do not increase radio complexity. The first algorithm uses OCs to cancel LI at the receiver. The second one uses the cross-correlation properties of the MCIDS spreading codes to combat RI.

Outer codes are a set of orthogonal sequences that duplicate MCIDS blocks. The set proposed in this design comprises of two sequences: $\{[+,+][+,-]\}$. The sequences are alternated at each node, starting with the first code at the source node.

Consider the nodes in Fig. 1. Node-B uses the second code to duplicate the MCIDS blocks before the shaping filter. The implication here is that the signal received at node- $\mathrm{B}$ duplicates MCIDS blocks according to the sequence $[+,+]$, whereas the LI duplicates them using the sequence $[+,-]$. Prior to despreading, the receiver adds together the duplicated MCIDS blocks, which cancels those that are duplicated locally. In the next node, the MCIDS blocks are not added but subtracted, given that the received blocks are spread with the $[+,-]$ outer code. The major drawback of this techinque is that it slows the transmission down. However, it provides an extra $3 \mathrm{~dB}$ gain for reception on top of LI cancellation.

The second algorithm exploits the low cross-correlation properties of the spreading sequences. Every transmitter delays its transmissions by $\frac{N \cdot T_{c}}{4}$ so that RI interferes with an orthogonal sequence located at $\frac{N \cdot T_{c}}{2}$ from the start of the first MCIDS block of the payload.

The aforementioned algorithms deal with signal cancellation. However, they do not account for the clipping produced by LI arrival. As the AGC is restricted to the packet preamble, which is LI free, the LNA scales the signal to the ADC voltage range. Upon LI arrival, the LNA saturates, and all information carried by the signal is lost. To reduce the resultant clipping effect, we delay transmissions by half $T_{c}$, so that LI appears 
in the second part of the chip, where little energy from the pulse arrives.

Clipping also occurs when RI interferes constructively, increasing the received power levels beyond those established by the AGC. A possible solution is to take into account RI and set a gain that prevents this form of clipping. In this way, we would still retain some of the information that is lost. However, this is not without cost since it increases ADC quantization errors due to the loss of resolution.

\section{PHY Layer protocol}

The PHY layer frame consists of 3 fields: preamble, header and payload. The preamble includes a Start Frame Delimiter (SFD) as well as a synchronization sequence. The header contains the PHY header, MAC header and the Header Check Sequence (HCS). Finally, the payload contains the data octets and a Frame Check Sequence (FCS). Also, Stuff Bits (SB) are appended to the data octets to fill the last MCIDS block.

The preamble is 8 MCIDS blocks in length, lasting $25 \mu \mathrm{s}$. The preamble synchronization sequence is 6 MCIDS blocks long. The SFD defines 3 radio operation modes: one for S\&F and two for cTxRx; each differs in the type of OC used during transmission.

The PHY header consists of three octets that define the scrambler seed identifier, the payload length in octets and its data rate. The radio has 4 possible working data rates: 7.5Mbps (BPSK), 15Mbps (QPSK), 30Mbps (16QAM) and 45Mbps (64QAM). The header has 40 MCIDS blocks, including the MAC header, and it is always transmitted at the nominal data rate $7.5 \mathrm{Mbps}$; the same data rate as the preamble.

\section{Performance}

In order to isolate the performance of the baseband algorithms, it is assumed that both the channel estimation and synchronization are perfect. Also, in order to minimize the interference produced by LI, the total duration of the UWB pulse is restricted to the first part of the chip, i.e., 8 samples lasting $3.125 \mathrm{~ns}$. However, the pulse width remains in the order of 2 ns.

The simulations are carried out using 100 channel impulse responses (CIRs) from each of the different multipath channel models (CM) specified by the 802.15.3a working group. These channel models are: CM1 for Line of Sight (LOS) and up to $4 \mathrm{~m}, \mathrm{CM} 2$ for NLOS and up to 4m, CM3 for NLOS and from $4 \mathrm{~m}$ to $10 \mathrm{~m}$ and CM4, which is the same as CM3 but with a RMS spread delay of $25 \mathrm{~ns}$.

As mentioned in Section II, LI presents a power level that is $20 \mathrm{~dB}$ below the transmission power and has no multipath component. The received signal and RI arrive with equal power as the nodes in the linear topology are assumed to be equidistant. However, both transmissions are subjected to different CIRs. All simulations run for 1000 packets; each 512 octets in size. We use the topology in Fig. 1 in our performance analysis, in particular node- $\mathrm{B}$, which is the worst case scenario.

Fig. 3 shows the simulation results for all channel models with the AGC switched off. In other words, the ADC has an infinite dynamic range, therefore no AGC is needed. All the cTxRx based curves are compared against their S\&F counterparts since the single-hop performance represents the upper bound for the cTxRx transceiver. Fig 3(a) shows that the transceiver works in all channel conditions. For CM4, the transceiver needs a minimum of $12 \mathrm{~dB}$ to ensure good reception. The cTxRx performance is only slightly worse compared to S\&F for all channels. The same applies for the QPSK results shown in Fig. 3(b).

In the case of Fig. 3(c) and Fig. 3(d), an error floor is observed. The error floor becomes more significant for 64QAM, where it increases with hasher channel conditions. The error floor is produced by RI propagating across different coded symbols due to multipath. The longer the multipath, the more acute the interference. Supporting this view are the results, see Fig. 3, for AWGN channel conditions for all modulation schemes, where both cTxRx and S\&F present the same performance curve.

In both Fig. 3(c) and Fig. 3(d), no communication is possible over CM3 and CM4. As a future work, a more effective cancellation algorithm for RI would be required to perform cTxRx at these rates and channel conditions. On the other hand, cTxRx and S\&F have the same performance at the BER range of $10^{-3}$ over CM1 and CM2. However, for Fig. 3(d), the performance over CM2 presents an error floor just above the maximum error rate line.

The results shown in Fig. 4 illustrate the effects of clipping. The curves labelled noagc refer to the functioning of an ideal ADC, whereas the ones labelled agc refer to the ADC being non-ideal given that an AGC is present. Clipping results in significant degradation across all channel models and data rates. Nevertheless, for BPSK and QPSK, as shown in Figure Fig. 4(a) and Fig. 4(b), communication remains viable in all channel conditions. In addition, as shown in Fig. 4(c), faster data rates are possible over CM1 and CM2. In the case of CM3 and CM4, the poor perfomance is mainly caused by the error floor instead of clipping. The similar performance shown in Fig. 4(c) for ideal and non-ideal ADC supports this fact. Similarly, for the 64QAM result shown in Fig. 4(d), communication is not possible due to the error floor.

\section{END-TO-End DATA RATES}

As mentioned in the introduction, cTxRx enables higher E2E data rates compared to the S\&F scenario. This section compares both approaches analytically. The E2E data rate is calculated for both scenarios assuming that no packets are lost at the relay nodes and the propagation time between nodes is negligible to the packet reception time at a given data rate.

We define the E2E data rate, $D_{e 2 e}$, as the ratio of the packet size in bits, $P_{b i t s}$, and the transmission time, $T_{\text {trans }}$. We define the transmission time as the time it takes to fully reach the receiver, with consideration for all relay nodes in the route.

Under the proposed cTxRx scenario, a relaying node starts transmitting after both the synchronization and header sections of the packet are received. In the ideal case, a relaying node does not incur such latency. However, in practice, a receiver needs to know when and what is being received.

To derive a general equation from which to infer both scenarios, we assumed that cTxRx uses no OC. Fig. 5 shows 


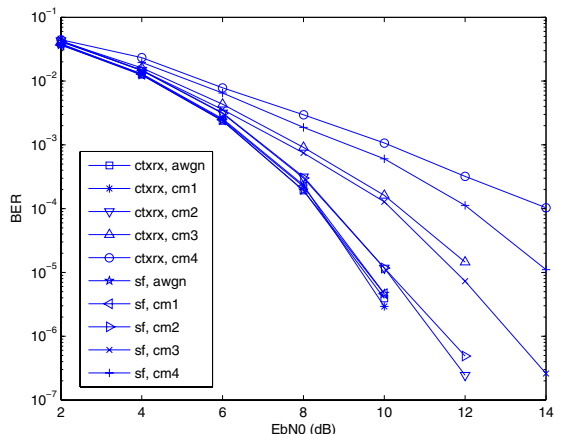

(a) BPSK

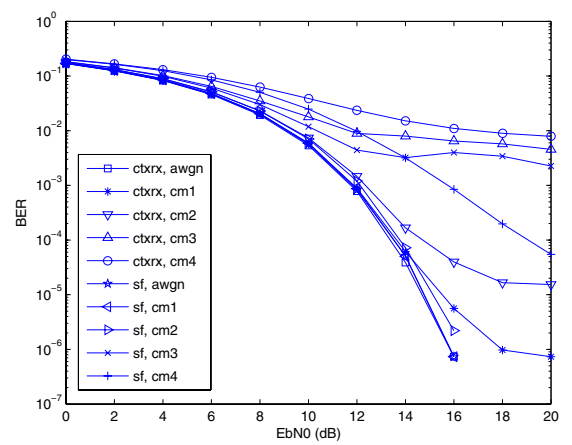

(c) 16QAM

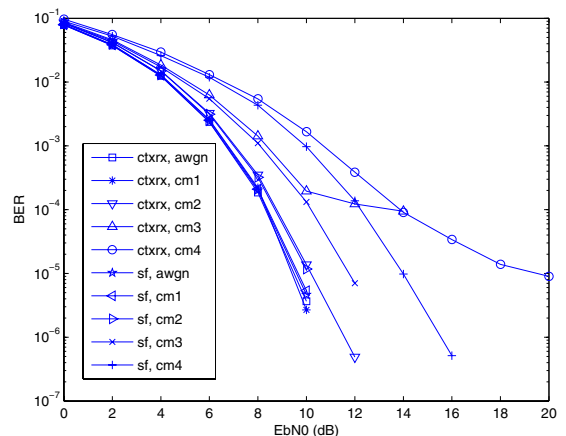

(b) QPSK

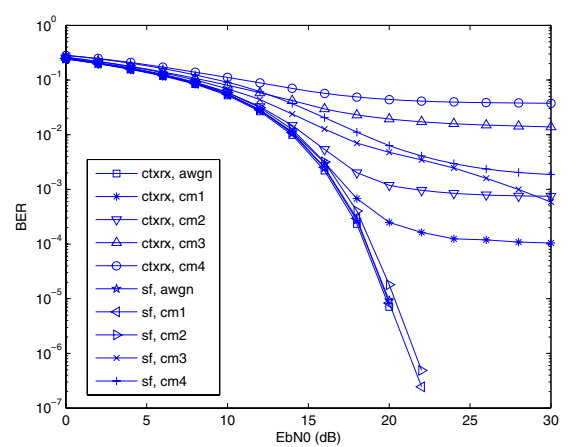

(d) 64QAM

Fig. 3. BER performance comparing cTxRx against S\&F.

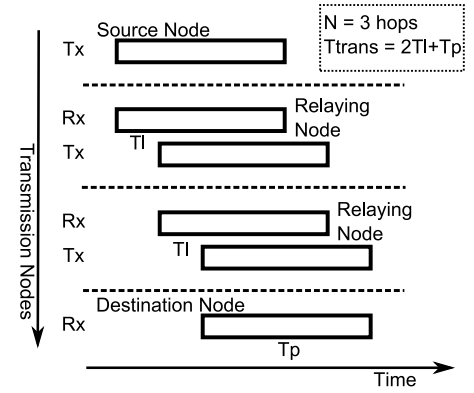

Fig. 5. cTxRx packet propagation.

the time line for the propagation of a packet over the transmission line depicted in Fig. 1. Every relay node presents a transmission latency, $T_{l}$, which contributes to the total transmission time.

To generalize the scenario in Fig. 5, we assume a transmission with $N$ hops, that is $N+1$ nodes including both the source and destination, and $N-1$ relaying nodes. The E2E data rate is given by (9).

$$
D_{e 2 e-\text { general }}=\frac{1}{1+(N-1) \cdot \frac{T_{l}}{T_{p}}}
$$

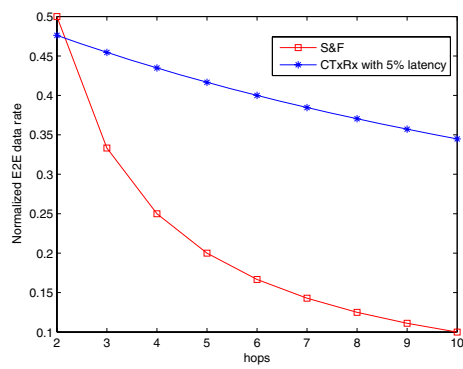

Fig. 6. CTxRx vs S\&F E2E maximum data rate comparison

$T_{p}$ is the time incurred by the relay nodes to receive a packet, which is the sum of $T_{l}$ and the time to transmit the payload. Also, (9) is normalized to the single-hop data rate, which is the maximum achievable data rate.

The proposed cTxRx system uses OC to prevent LI. We can safely extend the usage of OC to the preamble as its size is much smaller than the header and the payload. As a result, OC spreading doubles the packet size, thus halving the E2E data rate derived in (9). In the case of $\mathrm{S} \& \mathrm{~F}, T_{l}=T_{p}$, which yields $D_{e 2 e}=\frac{1}{N}$ when substituted in (9).

Fig. 6 compares cTxRx and S\&F transmissions over multiple relay nodes; i.e., $n \geq 2$, and a latency to packet time 


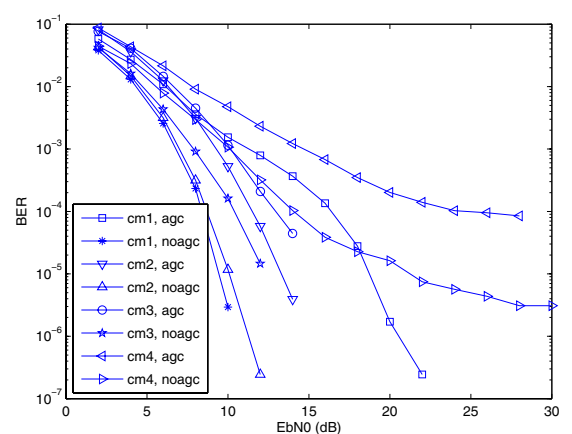

(a) BPSK

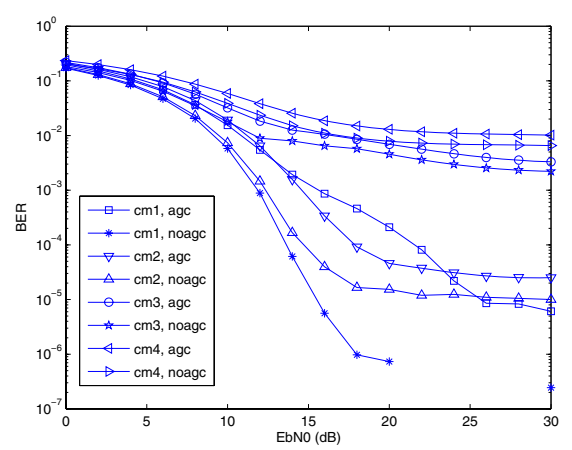

(c) 16QAM

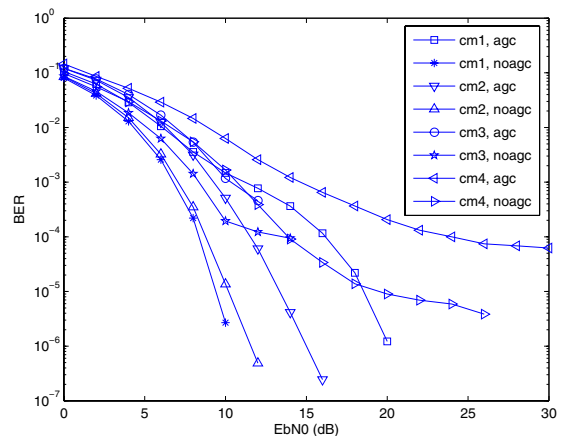

(b) QPSK

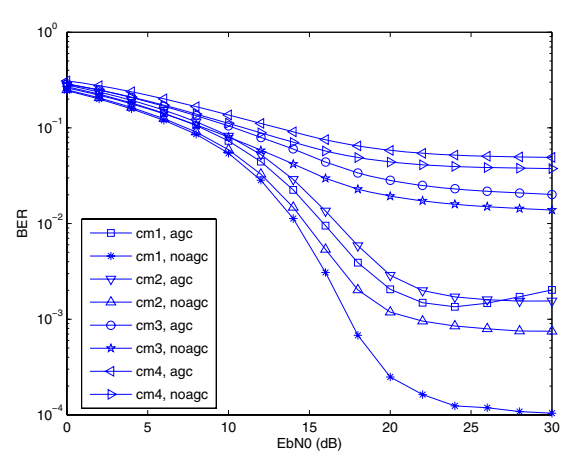

(d) 64QAM

Fig. 4. BER performance for non ideal AGC.

ratio of $\frac{T_{l}}{T_{p}}=0.05$. It shows that the maximum E2E data rate achievable for the cTxRx scenario is clearly better than that of the $S \& F$ scenario.

$$
\frac{T_{l}}{T_{p}}<\frac{N-2}{2 \cdot(N-1)}
$$

Another issue is the significance of the packet overhead with respect to the packet size, i.e., the $\frac{T_{l}}{T_{p}}$ ratio. If the overhead is large, it is better to transmit in $\mathrm{S} \& \mathrm{~F}$ mode. Eq. (10) relates the number of hops to the packet overhead, which shows that with increasing hops, a larger packet overhead can be accomodated, or conversely, a smaller packet payload can be transmitted.

\section{CONCLUSION}

In this paper, we have presented an UWB PHY capable of maintaining high data rates across a multihop mesh network. The transceiver works in cTxRx in order to maintain the single-hop transmission rate at the E2E level, which creates a novel channel model with increased interference. The channel model has been explained and the algorithms used to compensate for the interference validated via simulations. Also, we have addressed issues related to clipping at the receiver and has proven that the cTxRx concept is viable under different multipath channel conditions. Lastly, we have shown analytically the E2E performance benefits of cTxRx over S\&F.

\section{REFERENCES}

[1] X. Huang, D. Lowe, R. Gandia, and E. Dutkiewicz, "An impulse ultrawideband system capable of concurrent transmission and reception, part i: Requirements and innovations." in International conference on communications, circuits and systems, June 2006.

[2] R. Ramanathan, "Challenges: A radically new architecture for next generation mobile ad hoc networks," in Proc. ACM MOBICOM 2005, Cologne, Germany, 2005.

[3] Z. Fan, "Multi-hop mesh networking for UWB-based 802.15.3 coverage extension," in International Conference on Advanced Information Networking and Applications, vol. 1, April 2006, pp. 920-925.

[4] Q. Wu, Y. Xiong, Q. Zhang, Z. Guo, X.-G. Xia, and Z. Li, "Joint routing and topology formation in multihop UWB networks," IEEE J. Select. Areas Commun., vol. 24, pp. 843-849, 2006.

[5] X. Huang, D. Lowe, R. Gandia, and E. Dutkiewicz, "An impulse ultrawideband system capable of concurrent transmission and reception, part ii: Design and performance." in International conference on communications, circuits and systems, June 2006.

[6] J. Foerster, "Channel modeling sub-committee report final," IEEE 802.15, Doc. P802.15-02/490r1-SG3a, Tech. Rep., February 2003.

[7] X. Huang and Y. Li, "The multicode interleaved DSSS system for high speed wireless digital communications," in IEEE Int. Conference on Commun., vol. 10, 2001, pp. 2990-2994. 